\title{
Finite time blow-up for the nonlinear Schrödinger equation in trapped dipolar quantum gases with arbitrarily positive initial energy
}

Yi Yuan ${ }^{1}$ and Binhua Feng ${ }^{1 *}$

${ }^{*}$ Correspondence: binhuaf@163.com

1 Department of Mathematics, Northwest Normal University, Lanzhou, China

\section{Springer}

\begin{abstract}
In this paper, we study the blow-up criterion for the following nonlinear Schrödinger equation arising in trapped dipolar quantum gases:

$$
i \partial_{t} u=-\frac{1}{2} \Delta u+a^{2}\left(x_{1}^{2}+x_{2}^{2}+x_{3}^{2}\right) u+\lambda_{1}|u|^{2} u+\lambda_{2}\left(K *|u|^{2}\right) u, \quad(t, x) \in\left[0, T^{*}\right) \times \mathbb{R}^{3} .
$$

When $a=0$ or $a \neq 0$, by constructing an invariant set, we establish a new blow-up criterion, which implies the existence of blow-up solutions with arbitrarily large initial energy. This result gives a positive answer to the problem left by Carles, Markowich, and Sparber (Nonlinearity 21:2569-2590, 2008).
\end{abstract}

Keywords: Nonlinear Schrödinger equation; Trapped dipolar quantum gases; Blow-up criterion

\section{Introduction}

In the recent years the so-called dipolar Bose-Einstein condensate, i.e., a condensate made out of particles possessing a permanent electric or magnetic dipole moment, has attracted much attention; see, e.g., [2, 3, 17, 22-24]. At a temperature much smaller than the critical temperature, it is well described by the wave function $u(t, x)$ whose evolution is governed by the three-dimensional (3D) Schrödinger equation, see, e.g., [2, 3, 24, 35]:

$$
i \hbar \partial_{t} u=-\frac{\hbar^{2}}{2 m} \Delta u+W(x) u+U_{0}|u|^{2} u+\left(V_{\operatorname{dip}} *|u|^{2}\right) u, \quad x \in \mathbb{R}^{3}, t>0
$$

where $t$ is time, $x=\left(x_{1}, x_{2}, x_{3}\right)^{T} \in \mathbb{R}^{3}$ is the Cartesian coordinates, $*$ denotes the convolution, $\hbar$ is the Planck constant, $m$ is the mass of a dipolar particle, and $W(x)=a^{2}\left(x_{1}^{2}+x_{2}^{2}+x_{3}^{2}\right)$ is an external trapping potential, where $a$ is the trapping frequency. $U_{0}=4 \pi \hbar^{2} a_{s} / m$ describes the local interaction between dipoles in the condensate with $a_{s}$ the s-wave scattering length (positive for repulsive interaction and negative for attractive interaction). The

(c) The Author(s) 2020. This article is licensed under a Creative Commons Attribution 4.0 International License, which permits use, sharing, adaptation, distribution and reproduction in any medium or format, as long as you give appropriate credit to the original author(s) and the source, provide a link to the Creative Commons licence, and indicate if changes were made. The images or other third party material in this article are included in the article's Creative Commons licence, unless indicated otherwise in a credit line to the material. If material is not included in the article's Creative Commons licence and your intended use is not permitted by statutory regulation or exceeds the permitted use, you will need to obtain permission directly from the copyright holder. To view a copy of this licence, visit http://creativecommons.org/licenses/by/4.0/. 
long-range dipolar interaction potential between two dipoles is given by

$$
V_{\mathrm{dip}}(x)=\frac{\mu_{0} \mu_{\mathrm{dip}}^{2}}{4 \pi} \frac{1-3 \cos ^{2} \theta}{|x|^{3}}, \quad x \in \mathbb{R}^{3},
$$

where $\mu_{0}$ is the vacuum magnetic permeability, $\mu_{\text {dip }}$ is the permanent magnetic dipole moment, and $\theta$ is the angle between $x \in \mathbb{R}^{3}$ and the dipole axis $\mathbf{n} \in \mathbb{R}^{3}$, with $|\mathbf{n}|=1$.

In order to simplify the mathematical analysis, we rescale (1.1) into the following dimensionless Schrödinger equation:

$$
\left\{\begin{array}{l}
i \partial_{t} u=-\frac{1}{2} \Delta u+W(x) u+\lambda_{1}|u|^{2} u+\lambda_{2}\left(K *|u|^{2}\right) u, \quad(t, x) \in\left[0, T^{*}\right) \times \mathbb{R}^{3}, \\
u(0, x)=u_{0}(x) .
\end{array}\right.
$$

To simplify notation, we assume $\mathbf{n}=(0,0,1)$. The dimensionless long-range dipolar interaction potential $K(x)$ then reads as follows:

$$
K(x)=\frac{x_{1}^{2}+x_{2}^{2}-2 x_{3}^{2}}{|x|^{5}}, \quad x \in \mathbb{R}^{3} .
$$

We focus on the case when $\lambda_{1}$ and $\lambda_{2} \in \mathbb{R}$ fulfill the following conditions:

$$
\lambda_{1}< \begin{cases}\frac{4}{3} \pi \lambda_{2}, & \text { if } \lambda_{2}>0 \\ -\frac{8}{3} \pi \lambda_{2}, & \text { if } \lambda_{2}<0\end{cases}
$$

These conditions, following the terminology introduced in [8], define the unstable regime.

Because of important applications of equation (1.3) in physics, it has received much attention both from physics (see $[11,26]$ ) and mathematics (see $[1-5,7,8,18,20,21,28]$ ). Carles, Markowich, and Sparber in [8] first studied the local well-posedness and proved that the solution $u(t)$ of (1.3) blows up in finite time in the unstable regime if the initial energy is small. Ma, Cao, and Wang in [20,21] studied the sharp thresholds of global existence and blow-up, and proved that blow-up may occur if the initial energy $E\left(u_{0}\right)<d$ for some $d>0$. When $W(x)=0$, Huang in [18] discussed the exact value of $d$ by using the precise characterization of ground states of (2.5). Similar sharp thresholds of global existence and blow-up for other kinds of nonlinear Schrödinger equations are pursued strongly in $[9,12-16,32,36-41]$.

To our knowledge, the key to showing the existence of blow-up solutions is to prove $J^{\prime \prime}(t)<0$ for all $t \in\left[0, T^{*}\right)$, where $J(t)=\int_{\mathbb{R}^{3}}|x|^{2}|u(t, x)|^{2} d x$. If the solution $u(t)$ blows up in finite time $T^{*}$, i.e., $\|\nabla u(t)\|_{L^{2}} \rightarrow \infty$ as $t \rightarrow T^{*}$, it follows from Lemma 2.2 that, for any large $E\left(u_{0}\right)>0$, there exists $\delta>0$ such that $J^{\prime \prime}(t) \leq 6 E\left(u_{0}\right)-\int_{\mathbb{R}^{3}}|\nabla u(t, x)|^{2} d x<0$ for all $t \in\left[T^{*}-\delta, T^{*}\right)$. Therefore, a natural and interesting question is whether there exist blowup solutions with arbitrarily large initial energy. In particular, this is an open problem presented by Carles, Markowich, and Sparber, see Remark 5.3 in [8]. This will be the focus of the present paper. In fact, similar problems have been studied for other kinds of nonlinear evolutional equations, e.g., semilinear pseudo-parabolic equations and nonlinear Klein-Gordon equations, see [6, 19, 25, 27, 29-31, 33, 34].

Motivated by the above paper, in this paper, by establishing a blow-up criterion and a rather delicate analysis, we derive the existence of blow-up solutions with arbitrarily large initial energy. In particular, our method holds in the cases of both $a=0$ and $a \neq 0$. 
Theorem 1.1 Let $a \in \mathbb{R}, \lambda_{1}, \lambda_{2} \in \mathbb{R}$ and satisfy assumption (1.4). Then, for any $\mu>0$, there exists $u_{0} \in \Sigma:=\left\{v \in H^{1}\right.$ and $\left.|x| v \in L^{2}\right\}$ such that $E\left(u_{0}\right)=\mu$ and the corresponding solution $u(t)$ of (1.3) blows up in finite time.

Notation. Throughout this paper, for notational convenience, we use the following notation:

$$
F(u):=-\lambda_{1} \int_{\mathbb{R}^{3}}|u(x)|^{4} d x-\lambda_{2} \int_{\mathbb{R}^{3}}\left(K *|u|^{2}\right)(x)|u(x)|^{2} d x \quad \text { for all } u \in H^{1} .
$$

Let $u(t)$ be the solution of (1.3), we denote

$$
J(t):=\int_{\mathbb{R}^{3}}|x|^{2}|u(t, x)|^{2} d x, \quad \theta(t):=\sqrt{J(t)}
$$

\section{Preliminaries}

In this section, we recall some preliminary results that will be used later. Firstly, let us recall the local theory for Cauchy problem (1.3) established in [8].

Lemma 2.1 ([8]) For $a, \lambda_{1}, \lambda_{2} \in \mathbb{R}, u_{0} \in X:=\left\{v \in H^{1}\right.$, and $\left.\int_{\mathbb{R}^{3}} W(x)|v(x)|^{2} d x<\infty\right\}$, there exists $T=T\left(\left\|u_{0}\right\|_{X}\right)$ such that (1.3) admits a unique solution $u \in C([0, T], X)$. Let $\left[0, T^{*}\right)$ be the maximal time interval on which the solution $u(t)$ is well defined, if $T^{*}<\infty$, then $\|u(t)\|_{X} \rightarrow \infty$ as $t \rightarrow T^{*}$. Moreover, the solution $u(t)$ enjoys conservation of mass and energy, i.e., $\|u(t)\|_{L^{2}}=\left\|u_{0}\right\|_{L^{2}}$ and $E(u(t))=E\left(u_{0}\right)$ for all $t \in\left[0, T^{*}\right)$, where $E(u(t))$ is defined by

$$
E(u(t))=\frac{1}{2}\|\nabla u(t)\|_{L^{2}}^{2}+\int_{\mathbb{R}^{3}} W(x)|u(t, x)|^{2} d x-\frac{1}{2} F(u(t)) .
$$

In order to prove the existence of blow-up solution, we need the following virial identity, which can be proved by a similar argument as that in [9].

Lemma 2.2 ([8]) Let $u_{0} \in \Sigma$ and $u(t)$ be the solution of (1.3). Then function $J(t)$ belongs to $C^{2}\left[0, T^{*}\right)$, and

$$
\begin{aligned}
& J^{\prime}(t)=2 \operatorname{Im} \int_{\mathbb{R}^{3}} x \bar{u}(t, x) \nabla u(t, x) d x, \\
& J^{\prime \prime}(t)=2\|\nabla u(t)\|_{L^{2}}^{2}-4 \int_{\mathbb{R}^{3}} W(x)|u(t, x)|^{2} d x-3 F(u(t)) .
\end{aligned}
$$

Next, we recall the sharp Gagliardo-Nirenberg type inequality established in [1].

Lemma 2.3 ([1]) Let $\lambda_{1}, \lambda_{2} \in \mathbb{R}$ and satisfy assumption (1.4). Then, for all $u \in H^{1}$,

$$
F(u)=-\lambda_{1} \int_{\mathbb{R}^{3}}|u(x)|^{4} d x-\lambda_{2} \int_{\mathbb{R}^{3}} K *|u(x)|^{2}|u(x)|^{2} d x \leq C_{\mathrm{opt}}^{\frac{3}{2}}\|\nabla u\|_{L^{2}}^{3}\|u\|_{L^{2}},
$$

where the sharp constant $C_{\mathrm{opt}}=\frac{F(Q)^{\frac{2}{3}}}{\|Q\|_{L^{2}}^{2 / 3}\|\nabla\|_{L^{2}}^{2}}$ and $Q$ is the ground state of the following elliptic equation:

$$
-\frac{1}{2} \Delta Q+Q+\lambda_{1}|Q|^{2} Q+\lambda_{2}\left(K *|Q|^{2}\right) Q=0 .
$$


Moreover, the following Pohozaev's identities hold true:

$$
\|\nabla Q\|_{L^{2}}^{2}=\frac{3}{2} F(Q)=6\|Q\|_{L^{2}}^{2}
$$

From (2.6) and (2.1), we can obtain the following useful result:

$$
E(Q)=\frac{1}{4} F(Q)+\int_{\mathbb{R}^{3}} W(x)|Q|^{2} d x
$$

Thus, $C_{\text {opt }}$ can be rewritten as

$$
C_{\mathrm{opt}}=\left(\frac{2}{3}\right)^{\frac{2}{3}} \frac{\left(6 E(Q)-6 \int_{\mathbb{R}^{3}} W(x)|Q|^{2} d x\right)^{-\frac{1}{3}}}{\|Q\|_{L^{2}}^{2 / 3}} .
$$

Lemma 2.4 ([10]) Let $a \in \mathbb{R}, \lambda_{1}, \lambda_{2} \in \mathbb{R}$ and satisfy assumption (1.4). Then, for any $u \in \Sigma$, it holds

$$
\left(\operatorname{Im} \int_{\mathbb{R}^{3}} x \bar{u} \nabla u d x\right)^{2} \leq \int_{\mathbb{R}^{3}}|x|^{2}|u|^{2} d x\left(\int_{\mathbb{R}^{3}}|\nabla u|^{2} d x-\frac{F(u)^{\frac{2}{3}}}{\mathcal{C}_{\text {opt }}\|u\|_{L^{2}}^{\frac{2}{3}}}\right) .
$$

The proof is similar to the one in [10], so we omit it.

\section{Invariant evolution flow}

In this section, we construct a set which is invariant under the flow generated by Cauchy problem (1.3).

Proposition 3.1 Let $a \in \mathbb{R}, \lambda_{1}, \lambda_{2} \in \mathbb{R}$ and satisfy assumption (1.4). Taking an initial data $u_{0} \in \Sigma$ such that $4 E\left(u_{0}\right)>\frac{8}{27 \mathcal{C}_{\mathrm{opt}}^{3}\left\|u_{0}\right\|_{L^{2}}^{2}}$ and $\theta^{\prime}(0) \leq-\sqrt{\frac{y_{m}}{2}}$, where $y_{m}=4 E\left(u_{0}\right)-\frac{8}{27 C_{\mathrm{opt}}^{3}\left\|u_{0}\right\|_{L^{2}}^{2}}$. Define the set $K$ by

$$
K=\left\{v \in H^{1}, F(v)>\frac{8}{27 \mathcal{C}_{\text {opt }}^{3}\|v\|_{L^{2}}^{2}}\right\} .
$$

If $u_{0} \in K$, then the corresponding solution $u(t) \in K$ for all $t \in\left[0, T^{*}\right)$.

Proof Let $u_{0} \in K$ and $u(t)$ be the corresponding solution of (1.3). We deduce from $u_{0} \in K$ and (2.1) that

$$
2\left\|\nabla u_{0}\right\|_{L^{2}}^{2}+4 \int_{\mathbb{R}^{3}} W(x)\left|u_{0}(x)\right|^{2} d x-3 F\left(u_{0}\right)<4 E\left(u_{0}\right)-\frac{8}{27 \mathcal{C}_{\mathrm{opt}}^{3}\left\|u_{0}\right\|_{L^{2}}^{2}} .
$$

In fact, with the notation of $y_{m}$ and $J^{\prime \prime}(0)$, formula (3.1) implies that $\frac{J^{\prime \prime}(0)}{2}<\frac{y_{m}}{2}$. This combined with the assumption $\theta^{\prime}(0) \leq-\sqrt{\frac{y_{m}}{2}}$ implies that

$$
\theta^{\prime \prime}(0)=\frac{1}{\theta(0)}\left(\frac{J^{\prime \prime}(0)}{2}-\left(\theta^{\prime}(0)\right)^{2}\right)<0
$$


This implies that there exists $t_{0}>0$ such that $\theta^{\prime \prime}(t)<0$ for all $t \in\left[0, t_{0}\right]$. We then deduce that $\theta^{\prime}(t)<\theta^{\prime}(0)<-\sqrt{\frac{y_{m}}{2}}$, and so

$$
\left(\theta^{\prime}(t)\right)^{2}>\frac{y_{m}}{2}, \quad t \in\left[0, t_{0}\right]
$$

Next, using (2.1), (2.3), and the conservation of energy, we derive that

$$
\begin{aligned}
& F(u(t))=4 E\left(u_{0}\right)-J^{\prime \prime}(t)-8 \int_{\mathbb{R}^{3}} W(x)|u(t, x)|^{2} d x, \\
& \|\nabla u(t)\|_{L^{2}}^{2}=6 E\left(u_{0}\right)-J^{\prime \prime}(t)-10 \int_{\mathbb{R}^{3}} W(x)|u(t, x)|^{2} d x .
\end{aligned}
$$

Applying (3.4), (3.5), conservation of mass, and the definition of $J^{\prime}(t)$ in (2.2), we deduce from Lemma 2.4 that

$$
\begin{aligned}
\left(J^{\prime}(t)\right)^{2} \leq & 4 J(t)\left\{6 E\left(u_{0}\right)-J^{\prime \prime}(t)-10 \int_{\mathbb{R}^{3}} W(x)|u(t, x)|^{2} d x\right. \\
& \left.-\frac{1}{\mathcal{C}_{\mathrm{opt}}\left\|u_{0}\right\|_{L^{2}}^{2 / 3}}\left(4 E\left(u_{0}\right)-J^{\prime \prime}(t)-8 \int_{\mathbb{R}^{3}} W(x)|u(t, x)|^{2} d x\right)^{\frac{2}{3}}\right\} \\
< & 4 J(t)\left\{6 E\left(u_{0}\right)-J^{\prime \prime}(t)-8 \int_{\mathbb{R}^{3}} W(x)|u(t, x)|^{2} d x\right. \\
& \left.-\frac{1}{\mathcal{C}_{\mathrm{opt}}\left\|u_{0}\right\|_{L^{2}}^{2 / 3}}\left(4 E\left(u_{0}\right)-J^{\prime \prime}(t)-8 \int_{\mathbb{R}^{3}} W(x)|u(t, x)|^{2} d x\right)^{\frac{2}{3}}\right\} .
\end{aligned}
$$

We consequently obtain

$$
\left(\theta^{\prime}(t)\right)^{2}=\frac{\left(J^{\prime}(t)\right)^{2}}{4 J(t)} \leq \eta\left(J^{\prime \prime}(t)+8 \int_{\mathbb{R}^{3}} W(x)|u(t, x)|^{2} d x\right),
$$

where

$$
\eta(y)=6 E\left(u_{0}\right)-y-\frac{\left(4 E\left(u_{0}\right)-y\right)^{\frac{2}{3}}}{\mathcal{C}_{\mathrm{opt}}\left\|u_{0}\right\|_{L^{2}}^{2 / 3}} .
$$

Now, we deduce from (3.3) and (3.6) that

$$
\eta\left(J^{\prime \prime}(t)+8 \int_{\mathbb{R}^{3}} W(x)|u(t, x)|^{2} d x\right)>\frac{y_{m}}{2}=\eta\left(y_{m}\right)
$$

for all $t \in\left[0, t_{0}\right]$. Thus $J^{\prime \prime}(t)+8 \int_{\mathbb{R}^{3}} W(x)|u(t, x)|^{2} d x \neq y_{m}$ for all $t \in\left[0, t_{0}\right]$. By continuity, it follows that $J^{\prime \prime}(t)+8 \int_{\mathbb{R}^{3}} W(x)|u(t, x)|^{2} d x<y_{m}$ for all $t \in\left[0, t_{0}\right]$, which, together with (2.3) and the definition of $y_{m}$, implies $F(u(t))>\frac{8}{27 \mathcal{C}_{\mathrm{opt}}^{3}\left\|u_{0}\right\|_{L^{2}}^{2}}$ for all $t \in\left[0, t_{0}\right]$. Moreover, $\theta^{\prime \prime}\left(t_{0}\right)=\frac{1}{\theta\left(t_{0}\right)}\left(\frac{I^{\prime \prime}\left(t_{0}\right)}{2}-\left(\theta^{\prime}\left(t_{0}\right)\right)^{2}\right)<\frac{-4 \int_{\mathbb{R}^{3}} W(x)\left|u\left(t_{0}, x\right)\right|^{2} d x}{\theta\left(t_{0}\right)} \leq 0$. Therefore, applying an elementary Bootstrap argument, we can obtain

$$
F(u(t))>\frac{8}{27 \mathcal{C}_{\mathrm{opt}}^{3}\left\|u_{0}\right\|_{L^{2}}^{2}}=\frac{8}{27 \mathcal{C}_{\mathrm{opt}}^{3}\|u(t)\|_{L^{2}}^{2}}
$$

for all $t \in\left[0, T^{*}\right)$. This completes the proof. 


\section{Existence of blow-up solutions}

In this section, we use the same notations as in Sect. 3 and prove Theorem 1.1. Firstly, we establish the following blow-up criterion for (1.3).

Theorem 4.1 Let $a \in \mathbb{R}, \lambda_{1}, \lambda_{2} \in \mathbb{R}$ and satisfy assumption (1.4). Assume that $u_{0} \in \Sigma$ such that $J^{\prime}(0) \leq 0$. If

$$
\begin{aligned}
& 4\left\|u_{0}\right\|_{L^{2}}^{2} E\left(u_{0}\right)>\|Q\|_{L^{2}}^{2} F(Q), \\
& \left\|u_{0}\right\|_{L^{2}}^{2} F\left(u_{0}\right)>\|Q\|_{L^{2}}^{2} F(Q), \\
& E\left(u_{0}\right)\left\|u_{0}\right\|_{L^{2}}^{2}\left(1-\frac{\left(J^{\prime}(0)\right)^{2}}{8 E\left(u_{0}\right) J(0)}\right) \leq\|Q\|_{L^{2}}^{2}\left(E(Q)-\int_{\mathbb{R}^{3}} W(x)|Q|^{2} d x\right),
\end{aligned}
$$

then the solution $u(t)$ of (1.3) blows up in finite time.

Proof Firstly, it follows from (2.7) and (4.1) that $\left\|u_{0}\right\|_{L^{2}}^{2} E\left(u_{0}\right)>\|Q\|_{L^{2}}^{2}\left(E(Q)-\int_{\mathbb{R}^{3}} W(x) \times\right.$ $\left.|Q|^{2} d x\right)$. On the other hand, by (2.8) and the definition of $y_{m}$, we can obtain

$$
\left\|u_{0}\right\|_{L^{2}}^{2}\left(E\left(u_{0}\right)-\frac{y_{m}}{4}\right)=\|Q\|_{L^{2}}^{2}\left(E(Q)-\int_{\mathbb{R}^{3}} W(x)|Q|^{2} d x\right)
$$

These imply that (4.1) is equivalent to $y_{m}>0$, and (4.3) is equivalent to

$$
\left(\theta^{\prime}(0)\right)^{2} \geq \eta\left(y_{m}\right)=\frac{y_{m}}{2}
$$

In addition, the assumption $J^{\prime}(0) \leq 0$ implies $\theta^{\prime}(0) \leq 0$. In view of (2.7) and (4.4), assumption (4.2) yields the estimate

$$
\left\|u_{0}\right\|_{L^{2}}^{2} F\left(u_{0}\right)>4\|Q\|_{L^{2}}^{2}\left(E(Q)-\int_{\mathbb{R}^{3}} W(x)|Q|^{2} d x\right)=4\left\|u_{0}\right\|_{L^{2}}^{2}\left(E\left(u_{0}\right)-\frac{y_{m}}{4}\right),
$$

which, together with (3.4), implies

$$
J^{\prime \prime}(0)+8 \int_{\mathbb{R}^{3}} W(x)\left|u_{0}(x)\right|^{2} d x<y_{m}
$$

Therefore, $F\left(u_{0}\right)>\frac{8}{27 \mathcal{C}_{\mathrm{opt}}^{3}\left\|u_{0}\right\|_{L^{2}}^{2}}$. It follows from Proposition 3.1 that $F(u(t))>\frac{8}{27 \mathcal{C}_{\mathrm{opt}}^{3}\left\|u_{0}\right\|_{L^{2}}^{2}}$ for all $t \in\left[0, T^{*}\right)$. Moreover, we know from the proof of Proposition 3.1 that $\left(\theta^{\prime}(t)\right)^{2}>\frac{y_{m}}{2}$ for all $t \in\left[0, T^{*}\right)$. We consequently obtain

$$
\theta^{\prime \prime}(t)=\frac{1}{\theta(t)}\left(\frac{J^{\prime \prime}(t)}{2}-\left(\theta^{\prime}(t)\right)^{2}\right)<0 \quad \text { for all } t \in\left[0, T^{*}\right)
$$

This implies that the solution $u(t)$ of (1.3) blows up in finite time. If not, i.e., $T^{*}=+\infty$, it follows from $\theta^{\prime}(0) \leq 0$ and (4.7) that

$$
\theta(t)=\theta(1)+\int_{1}^{t} \theta^{\prime}(s) d s<\theta(1)+\theta^{\prime}(1)(t-1)<0
$$

for sufficiently large $t$, which is a contradiction with $\theta(t)>0$ for all $t \in\left[0, T^{*}\right)$. 
Finally, we give the proof of Theorem 1.1.

Proof of Theorem 1.1 We claim that the result in Theorem 4.1 can be valid for arbitrarily large energy. Let $v_{0}=\gamma^{2} Q(\gamma x)$ for $\gamma>0$. By some simple calculations, we have

$$
\left\|v_{0}\right\|_{L^{2}}^{2}=\gamma\|Q\|_{L^{2}}^{2}, \quad F\left(v_{0}\right)=\gamma^{5} F(Q),
$$

and

$$
E\left(v_{0}\right)=\frac{\gamma^{3}}{2}\|\nabla Q\|_{L^{2}}^{2}+\frac{1}{\gamma} \int_{\mathbb{R}^{3}} W(x)|Q|^{2} d x-\frac{\gamma^{5}}{2} F(Q) .
$$

These yield

$$
4\left\|v_{0}\right\|_{L^{2}}^{2} E\left(v_{0}\right) \leq\|Q\|_{L^{2}}^{2} F(Q) \quad \text { and } \quad\left\|v_{0}\right\|_{L^{2}}^{2} F\left(v_{0}\right)>\|Q\|_{L^{2}}^{2} F(Q)
$$

for sufficiently large $\gamma>0$.

Let $u_{0}(x)=e^{i \lambda|x|^{2}} v_{0}(x)$ with $\lambda<0$ and $u(t)$ be the corresponding solution of (1.3). It easily follows that

$$
\begin{aligned}
& \left\|u_{0}\right\|_{L^{2}}^{2}=\left\|v_{0}\right\|_{L^{2}}^{2} \quad \text { and } \quad E\left(u_{0}\right)=E\left(v_{0}\right)+2 \lambda^{2}\left\|x v_{0}\right\|_{L^{2}}^{2}+2 \lambda \operatorname{Im} \int_{\mathbb{R}^{3}} \bar{v}_{0} x \cdot \nabla v_{0} d x, \\
& \operatorname{Im} \int_{\mathbb{R}^{3}} \bar{u}_{0} x \cdot \nabla u_{0} d x=\operatorname{Im} \int_{\mathbb{R}^{3}} \bar{v}_{0} x \cdot \nabla v_{0} d x+2 \lambda\left\|x v_{0}\right\|_{L^{2}}^{2} .
\end{aligned}
$$

Combining (4.9) and (4.10), we further infer

$$
E\left(u_{0}\right)-\frac{\left(\operatorname{Im} \int_{\mathbb{R}^{3}} \bar{u}_{0} x \cdot \nabla u_{0} d x\right)^{2}}{2\left\|x u_{0}\right\|_{L^{2}}^{2}}=E\left(v_{0}\right)-\frac{\left(\operatorname{Im} \int_{\mathbb{R}^{3}} \bar{v}_{0} x \cdot \nabla v_{0} d x\right)^{2}}{2\left\|x v_{0}\right\|_{L^{2}}^{2}},
$$

which implies (4.3). In addition, due to (4.8), the estimate (4.2) also holds. Choosing sufficiently small $\lambda \in(-\infty, 0)$, we deduce from formulas (4.9) and (4.10) that

$$
4\left\|u_{0}\right\|_{L^{2}}^{2} E\left(u_{0}\right)>\|Q\|_{L^{2}}^{2} F(Q) \text { and } \operatorname{Im} \int_{\mathbb{R}^{3}} x \bar{u}_{0} \nabla u_{0} d x<0 .
$$

Applying Theorem 4.1, the solution $u(t)$ of (1.3) with initial data $u_{0}$ blows up in finite time. On the other hand, we see from (4.9) that $E\left(u_{0}\right) \rightarrow+\infty$ as $\lambda \rightarrow-\infty$. Therefore, the initial energy can be arbitrarily large. This completes the proof.

\section{Conclusions}

In this paper, we study the existence of blow-up solutions for the nonlinear Schrödinger equation arising in trapped dipolar quantum gases with arbitrarily large initial energy. We consider two cases, one is that the system is free, the other is that a harmonic potential is added. In both cases, by constructing an invariant set, we establish a new blow-up criterion, which implies the existence of blow-up solutions with arbitrarily large initial energy. 
Funding

This paper is supported by the National Natural Science Foundation of China (No. 11601435).

\section{Abbreviations}

Not applicable.

Availability of data and materials

Not applicable.

\section{Competing interests}

The authors declare that no competing interests exist.

\section{Authors' contributions}

The authors contributed equally to this paper. All authors read and approved the final manuscript.

\section{Publisher's Note}

Springer Nature remains neutral with regard to jurisdictional claims in published maps and institutional affiliations.

Received: 9 January 2020 Accepted: 10 March 2020 Published online: 18 March 2020

\section{References}

1. Antonelli, P., Sparber, C.: Existence of solitary waves in dipolar quantum gases. Physica D 240, 426-431 (2011)

2. Bao, W., Cai, Y.: Mathematical theory and numerical methods for Bose-Einstein condensation. Kinet. Relat. Models 6 , $1-135$ (2013)

3. Bao, W., Cai, Y., Wang, H.: Efficient numerical methods for computing ground states and dynamics of dipolar Bose-Einstein condensates. J. Comput. Phys. 229, 7874-7892 (2010)

4. Bellazzini, J., Forcella, L.: Asymptotic dynamic for dipolar quantum gases below the ground state energy threshold. J. Funct. Anal. 277, 1958-1998 (2019)

5. Bellazzini, J., Jeanjean, L.: On dipolar quantum gases in the unstable regime. SIAM J. Math. Anal. 48, 2028-2058 (2016)

6. Cao, J., Song, G., Wang, J., Shi, Q., Sun, S.: Blow-up and global solutions for a class of time fractional nonlinear reaction-diffusion equation with weakly spatial source. Appl. Math. Lett. 91, 201-206 (2019)

7. Carles, R., Hajaiej, H.: Complementary study of the standing wave solutions of the Gross-Pitaevskii equation in dipolar quantum gases. Bull. Lond. Math. Soc. 47, 509-518 (2015)

8. Carles, R., Markowich, P.A., Sparber, C.: On the Gross-Pitaevskii equation for trapped dipolar quantum gases. Nonlinearity 21, 2569-2590 (2008)

9. Cazenave, T.: Semilinear Schrödinger Equations. Courant Lecture Notes in Mathematics, vol. 10. New York University, Courant Institute of Mathematical Sciences, New York; American Mathematical Society, Providence (2003)

10. Duyckaerts, T., Roudenko, S.: Going beyond the threshold: scattering and blow-up in the focusing NLS equation. Commun. Math. Phys. 344, 1573-1615 (2015)

11. Ellio, M.S., Valentini, J.J., Chandler, D.W.: Subkelvin cooling NO molecules via "billiard-like" collisions with argon. Science 302, 1940-1943 (2003)

12. Feng, B.: Sharp threshold of global existence and instability of standing wave for the Schrödinger-Hartree equation with a harmonic potential. Nonlinear Anal., Real World Appl. 31, 132-145 (2016)

13. Feng, B.: On the blow-up solutions for the nonlinear Schrödinger equation with combined power-type nonlinearities J. Evol. Equ. 18, 203-220 (2018)

14. Feng, B.: On the blow-up solutions for the fractional nonlinear Schrödinger equation with combined power-type nonlinearities. Commun. Pure Appl. Anal. 17, 1785-1804 (2018)

15. Feng, B., Chen, R., Wang, Q.: Instability of standing waves for the nonlinear Schrödinger-Poisson equation in the $L^{2}$-critical case. J. Dyn. Differ. Equ. (2019). https://doi.org/10.1007/s10884-019-09779-6

16. Feng, B., Liu, J., Niu, H., Zhang, B.: Strong instability of standing waves for a fourth-order nonlinear Schrödinge equation with the mixed dispersions. Nonlinear Anal. 196, 111791 (2020)

17. Gligoric, G., Maluckov, A., Sterpic, M., Hadzievski, I., Malomed, B.A.: Two dimensional discrete solutions in dipolar Bose-Einstein condensates. Phys. Rev. A 81, 13633 (2010)

18. Huang, J., Zhang, J.: Exact value of cross-constrain problem and strong instability of standing waves in trapped dipolar quantum gases. Appl. Math. Lett. 70, 32-38 (2017)

19. Lian, W., Ahmed, M., Xu, R.: Global existence and blow up of solution for semilinear hyperbolic equation with logarithmic nonlinearity. Nonlinear Anal. 184, 239-257 (2019)

20. Ma, L., Cao, P.: The threshold for the focusing Gross-Pitaevskii equation with trapped dipolar quantum gases. J. Math. Anal. Appl. 381, 240-246 (2011)

21. Ma, L., Wang, J.: Sharp threshold of the Gross-Pitaevskii equation with trapped dipolar quantum gases. Can. Math. Bull. 56(2), 378-387 (2013)

22. Nath, R., Pedri, P., Santos, I.: Soliton-soliton scattering in dipolar Bose-Einstein condensates. Phys. Rev. A 76, 013606 (2007)

23. Pedri, P., Santos, l.: Two-dimensional bright solitons in dipolar Bose-Einstein condensates. Phys. Rev. Lett. 95, 200404 (2005)

24. Santos, L., Shlyapnikov, G., Zoller, P., Lewenstein, M.: Bose-Einstein condensation in trapped dipolar gases. Phys. Rev. Lett. 85, 1791-1797 (2000)

25. Shen, J., Yang, Y., Chen, S., Xu, R.: Finite time blow up of fourth-order wave equations with nonlinear strain and source terms at high energy level. Int. J. Math. 24, 1350043 (2013)

26. Vengalattore, M., Leslie, S.R., Guzman, J., Stamper-Kurn, D.M.: Spontaneously modulated spin textures in a dipolar spinor Bose-Einstein condensate. Phys. Rev. Lett. 100, 170403 (2008) 
27. Xiang, M., Radulescu, V.D., Zhang, B.: Nonlocal Kirchhoff diffusion problems: local existence and blow-up of solutions. Nonlinearity 31, 3228-3250 (2018)

28. Xie, Y., Li, L., Zhu, S.: Dynamical behaviors of blowup solutions in trapped quantum gases: concentration phenomenon. J. Math. Anal. Appl. 468, 169-181 (2018)

29. Xu, R., Su, J.: Global existence and finite time blow-up for a class of semilinear pseudo-parabolic equations. J. Funct. Anal. 264, 2732-2763 (2013)

30. Xu, R., Wang, X., Yang, Y.: Blowup and blowup time for a class of semilinear pseudo-parabolic equations with high initial energy. Appl. Math. Lett. 83, 176-181 (2018)

31. Xu, R., Wang, X., Yang, Y., Chen, S.: Global solutions and finite time blow-up for fourth order nonlinear damped wave equation. J. Math. Phys. 59, 061503 (2018)

32. Yang, L., Li, X., Wu, Y., Caccetta, L.: Global well-posedness and blow-up for the Hartree equation. Acta Math. Sci. Ser. B Engl. Ed. 37, 941-948 (2017)

33. Yang, Y., Xu, R.: Finite time blowup for nonlinear Klein-Gordon equations with arbitrarily positive initial energy. Appl. Math. Lett. 77, 21-26 (2018)

34. Yang, Y., Xu, R.: Nonlinear wave equation with both strongly and weakly damped terms: supercritical initial energy finite time blow up. Commun. Pure Appl. Anal. 18, 1351-1358 (2019)

35. Yi, S., You, L.: Trapped atomic condensates with anisotropic interactions. Phys. Rev. A 61, 041604 (2000)

36. Yue, Z., Li, X., Zhang, J.: A new blow-up criterion for Gross-Pitaevskii equation. Appl. Math. Lett. 62, 16-22 (2016)

37. Zhang, J: Sharp conditions of global existence for nonlinear Schrödinger and Klein-Gordon equations. Nonlinear Anal. 48, 191-207 (2002)

38. Zhang, J.: Sharp threshold for blowup and global existence in nonlinear Schrödinger equations under a harmonic potential. Commun. Partial Differ. Equ. 30, 1429-1443 (2005)

39. Zhang, J., Zhu, S.: Sharp blow-up criteria for the Davey-Stewartson system in $\mathbb{R}^{3}$. Dyn. Partial Differ. Equ. 8, 239-260 (2011)

40. Zhang, J., Zhu, S.: Sharp energy criteria and singularity of blow-up solutions for the Davey-Stewartson system. Commun. Math. Sci. 17, 653-667 (2019)

41. Zhu, S.: On the Davey-Stewartson system with competing nonlinearities. J. Math. Phys. 57, 031501 (2016)

\section{Submit your manuscript to a SpringerOpen ${ }^{\circ}$ journal and benefit from:}

- Convenient online submission

- Rigorous peer review

- Open access: articles freely available online

- High visibility within the field

- Retaining the copyright to your article

Submit your next manuscript at $\boldsymbol{~ s p r i n g e r o p e n . c o m ~}$ 